\title{
Posttranslational Activation of Endothelial Nitric Oxide Synthase Attenuates Carbon Tetrachloride-Induced Hepatotoxicity in Newborn Rats
}

\author{
HUA LIU, CHARU SUBRAMONY, AND MICHAEL NOWICKI \\ Department of Pediatrics [H.L., M.N.], Department of Pathology [C.S.], University of Mississippi Medical Center, Jackson, \\ Mississippi 36216
}

\begin{abstract}
Nitric Oxide (NO) can be cytotoxic or cytoprotective depending on amount and location of its generation. eNOS is important in modulating blood flow and is allosterically regulated. Inducible NOS (iNOS) tends to produce large quantities of NO leading to cell injury. We studied the role and regulation of NOS in carbon tetrachloride $\left(\mathrm{CCl}_{4}\right)$-induced hepatotoxicity in newborn rats. eNOS was expressed before birth, significantly increased on day of life (DOL) 2 reaching a maximum at DOL-20. iNOS was absent at all ages. $\mathrm{CCl}_{4}$ treatment resulted in hepatic injury in newborn rats and damage was intensified by co-administration of a general NOS inhibitor. $\mathrm{CCl}_{4}$ treatment increased eNOS activity without change in mRNA or protein levels. Administration of $\mathrm{CCl}_{4}$ resulted in an increase in phosphorylation of threonine protein kinase (Akt) and eNOS, associated with an increase in eNOS activity. Administration of wortmannin (phosphatidylinositol 3-kinase, PI3 K, inhibitor) attenuated the phosphorylation of Akt and eNOS and reduced eNOS activity. Co-administration of $\mathrm{CCl}_{4}$ and wortmannin potentiated the degree of hepatic injury. iNOS was not detectable in $\mathrm{CCl}_{4}$-treated rats. This data indicates a protective role for eNOS in $\mathrm{CCl}_{4}$-induced hepatotoxicity in newborn rats with protection accomplished by activation of eNOS via posttranslational modification of the PI3 K/Akt signaling pathway. (Pediatr Res 63: 613-619, 2008)
\end{abstract}

$\mathrm{D}$ ifferences exist in the response to toxic hepatic injury in neonates compared with adults. Previous studies have indicated that immature liver is partially protected from carbon tetrachloride $\left(\mathrm{CCl}_{4}\right)$-induced injury (1-3); proposed mechanisms include an underdeveloped cytochrome P-450 system and increased stimulation of hepatocellular regeneration and tissue repair mechanisms $(1,4)$. Newer evidence shows that acute $\mathrm{CCl}_{4}$ toxicity increases nitric oxide synthase (NOS) activity resulting in increased hepatic blood flow, both in the portal vein and hepatic artery $(5,6)$. Co-administration of $\mathrm{CCl}_{4}$ and NOS inhibitors dampens increased hepatic artery blood flow, worsening of hepatocellular damage $(5,6)$, suggesting a protective role for NOS in this model via increased hepatic artery blood flow.

Hepatic blood flow in neonates differs from adults by nature of a ductus venosus, which shunts blood away from hepatocytes. There are also maturational differences in hepatic blood

Received October 10, 2007; Accepted: January 25, 2008

Correspondence: Michael J. Nowicki, M.D., Division of Pediatric Gastroenterology, University of Mississippi Medical Center, 2500 North State Street, Jackson, MS 36216; e-mail: mnowicki@ped.umsmed.edu

Supported by a Department Research Grant. flow regulation; the hepatic artery contributes more to maintenance of sinusoidal blood flow in the immature liver than in the adult liver (7). Normally, hepatic blood flow is under the control of eNOS present in sinusoidal endothelial cells (8). Moreover, there is developmental expression of NOS in the liver. eNOS is minimally expressed in the prenatal liver, increases rapidly after birth, and reaches a maximum at $20 \mathrm{~d}$ of life, which is maintained in adults (9). Hepatic iNOS activity is not detectable during normal development (9).

Nitric oxide (NO) plays important roles in the liver with potential for being both cytotoxic and cytoprotective. Factors in determining beneficial versus harmful effects include location, amount, and duration of NO generation. eNOS is constitutively expressed in the endothelium of most tissues, playing an important role in regulating blood flow in response to hemodynamic shear stress and endothelial-dependent vasodilators. NO generated by eNOS is allosterically regulated and in small amounts leads to vasodilation. Unlike eNOS, iNOS is inducible and present in most liver cells. iNOS lacks fine regulation and tends to produce large quantities of NO once induced. Excessive NO leads to cell injury through nitrosylation of cellular enzymes and potentiation of oxidative stress by forming peroxynitrite (reviewed in Ref. 10). Normally only eNOS is present in the liver, whereas hepatic iNOS is upregulated by a number of conditions (endotoxemia, hemorrhagic shock, sepsis, ischemia-reperfusion, and liver regeneration). NO has been shown to protect against oxidative damage and peroxidation induced by experimental models such as alcohol (11), $\mathrm{H}_{2} \mathrm{O}_{2}$-mediated oxidative stress (12), and $\mathrm{CCl}_{4}$ (13).

The regulation and roles of eNOS and iNOS in $\mathrm{CCl}_{4}$ induced hepatotoxicity have not been elucidated in the developing liver. We performed this study to determine what role age-related differences in NOS expression plays in acute $\mathrm{CCl}_{4}$-induced hepatocellular injury.

\section{METHODS}

Chemicals. Unless otherwise stated, all chemicals in this study were purchased from Sigma Chemical Co. (St. Louis, MO). Antibodies for Western

Abbreviations: Akt, serine/threonine protein kinase or protein kinase B; ALT, alanine aminotransferase; BW, body weight; DOL, day of life; LNAME, L-N (G)-nitroarginine methyl ester; MDA, malondialdehyde; PI3K, phosphatidylinositol 3-kinase; ROS, reactive oxygen species 
blot were purchased as follows: anti-eNOS and anti-iNOS from BD Biosciences (San Diego, CA), antiactin from Chemicon (Temecula, CA), antiphospho-eNOS, antiserine/threonine protein kinase (Akt), antiphospho-Akt, and wortmannin (a phosphatidylinositol 3-kinase, PI3 K, inhibitor) were purchased from Cell Signaling (Danvers, MA).

Animal treatment. Pregnant Sprague-Dawley rats were obtained at $14 \mathrm{~d}$ of gestation and kept in plastic cages with free access to food and water with alternating 12-h periods of light and darkness. In experiments requiring fetal tissue, the dam was killed by $\mathrm{CO}_{2}$ narcosis and cervical dislocation, followed by delivery of fetuses by cesarean section. In age-related experiments, newborn rats were kept with the mother with free access to breast-feeding before weaning, and standard rat chow and water post weaning. The age of rats studied were day of life (DOL) 0, 2, 5, 10, and 20. Adult, male SpragueDawley rats $(200-250 \mathrm{~g})$ were used as the adult time point, to avoid any potential effect of estrous on NOS expression. In the study of $\mathrm{CCl}_{4}$-induced hepatotoxicity, pups of either sex from various litters of equal postnatal age were randomized to one of the three experimental groups: 1) control group (vehicle, corn oil), 2) $\mathrm{CCl}_{4}[100 \mu \mathrm{L} / \mathrm{kg}$ body weight (BW), i.p.], or 3) L-N(G)-nitroarginine methyl ester (L-NAME, $2 \mathrm{mg} / \mathrm{kg} \mathrm{BW}$, i.p.) $1 \mathrm{~h}$ before $\mathrm{CCl}_{4}$ injection. Animals were killed $24 \mathrm{~h}$ following injection. For study of posttranslational regulation, DOL-20 pups were randomly divided into four experimental groups: 1) control group (vehicle, corn oil), 2) $\mathrm{CCl}_{4}(100 \mu \mathrm{L} / \mathrm{kg}$ BW, i.p.), 3) wortmannin (1.4 mg/kg BW, i.p.) $1 \mathrm{~h}$ before and $12 \mathrm{~h}$ after $\mathrm{CCl}_{4}$ injection, or 4) wortmannin alone (1.4 mg/kg BW, i.p.). Animals were killed $24 \mathrm{~h}$ after injection. The study was approved by the Institutional Animal Care and Use Committee at the University of Mississippi Medical Center.

Evaluation of $\mathrm{CCl}_{4}$ hepatotoxicity. Blood samples were collected and centrifuged at room temperature for $10 \mathrm{~min}$ at $13,000 \mathrm{rpm}$. Serum was assayed for alanine aminotransferase (ALT) activity following the protocol of an ALT assay kit (Sigma Chemical Co. Diagnostics, St. Louis, MO). Liver samples from each aged animal were fixed in $10 \%$ formalin, paraffin-embedded, and sections obtained and stained with hemotoxylin and eosin for histologic evaluation.

Determination of lipid peroxidation. Tissue concentration of malondialdehyde (MDA), an intermediate of lipid peroxidation, was measured as described previously (14).

Real time RT-PCR for NOS. cDNA was synthesized using iScript cDNA Synthesis system (Bio-Rad Hercules, CA) on total cellular RNA. Controls without reverse transcriptase were performed for each sample to ensure absence of genomic DNA. Real time PCR was carried out in a real time thermal cycler (iCycler, Bio-Rad) using iQ SYBR Green Supermix (BioRad). Cycling conditions were $5 \mathrm{~min}$ at $95^{\circ} \mathrm{C}$, followed by 40 cycles of $15 \mathrm{~s}$ at $95^{\circ} \mathrm{C}, 15 \mathrm{~s}$ at $55^{\circ} \mathrm{C}$, then $60 \mathrm{~s}$ at $72^{\circ} \mathrm{C}$. PCR specificity was tested via analysis of the melting curve and agarose gel electrophoresis. To semiquantify input amounts of templates, standard curves were constructed with serial dilutions of cDNA sample from a positive control (brain tissue from lipopolysaccharide-treated rat). To standardize results, interpolated values for each sample were divided by the value of the housekeeping gene glyceraldehyde-3-phosphate dehydrogenase (GAPDH). Primers were designed with Primer3 software (15) and checked for absence of crossreactivity by BLAST search. The primer pairs used, product size, and positive controls are shown in Table 1 .

Western blotting for eNOS, iNOS, and Akt. Liver tissue was homogenized in $50 \mathrm{mM}$ HEPES, pH 7.4, $1 \mathrm{mM}$ EDTA and protease inhibitor cocktail, followed by centrifugation at $15,000 \mathrm{~g}$ for $30 \mathrm{~min}$. Protein was separated by $7.5 \%$ SDS-PAGE, transferred to nitrocellulose membranes, and visualized by Enhanced Chemiluminescence System (Amersham Health). Densitometric quantification of Western blot signal intensity of autoradiograms was performed using a Molecular Dynamics P.D densitometer.

Measurement of eNOS and iNOS activity. NOS activity was estimated by formation of nitrite/nitrate from L-arginine through the in vitro reaction of NOS, according to the method described by Kelley et al. (16). Nitrite/ nitrate generated in the reaction mixture by NOS activity was measured with a Nitric Oxide Synthase Assay kit (Calbiochem, San Diego, CA). An eNOS inhibitor L-N (5)-(1-iminoethyl)-ornithine (L-NIO) at final concen- tration of $10 \mu \mathrm{M}$ in the reaction mixture was used in a separate set of eNOS activity assays to confirm eNOS specific activity.

Statistical analysis. Values are expressed as mean \pm SE (SEM). Statistical analysis was performed using unpaired $t$ test (for only two groups) and analysis of variance (for more than two groups). Statistical significance was considered at $p<0.05$.

\section{RESULTS}

Age related eNOS and iNOS expression. Ontogeny of hepatic eNOS, as determined by western blot analysis and activity (Fig. $1 A$ and $B$ ), was similar to our previous study (9). eNOS was presented before birth, albeit at low levels. On DOL-2 eNOS protein and activity were significantly increased compared with earlier times. There was further increase until a maximum was attained at DOL-20, which persisted in the adult. NOS mRNA was determined by real time RT-PCR. Validation of the PCR and primers was performed by serial dilution of cDNA. The coefficiency of the PCR was $>0.995$ and the efficiency of the PCR was $100 \pm 10 \%$. eNOS mRNA showed a pattern similar to its protein expression, with the exception of a relatively lower level of expression in the adult (Fig. 1C). iNOS mRNA, protein, and activity were undetectable at all ages.

Hepatic damage due to $\boldsymbol{C C l}_{4}$. Hepatic injury, measured by serum ALT, was noted in all ages (DOL-0 to DOL-20, Fig. $2 A)$. ALT increase was mild; about 3-fold over control values. Co-administration of $\mathrm{CCl}_{4}$ and the nonselective NOS inhibitor L-NAME increased hepatic damage compared with $\mathrm{CCl}_{4}$ alone starting on DOL-2 (Fig. 2A). Administration of L-NAME alone had no effect on ALT $(16.69 \pm 3.88 \mathrm{U} / \mathrm{mL})$. Histologic examination showed typical features of $\mathrm{CCl}_{4}$ toxicity from DOL-2 to adult (Fig. $3 C$ and $D$ ), such as ballooning degeneration with pycnotic nuclei and individual hepatocyte necrosis; fibrosis was not seen. In adults, the findings were most pronounced in zone 3 (central vein), with some involvement of zone 2. In less mature animals, histologic features were found throughout the hepatic lobule, although with a prominence in zone 3. Animals exposed to $\mathrm{CCl}_{4}$ on DOL-0 had minimal evidence of injury, limited to scattered necrotic hepatocytes (Fig. 3B).

Determination of lipid peroxidation. Determination of MDA showed an increase in lipid peroxidation following administration of $\mathrm{CCl}_{4}$ (Fig. $2 \mathrm{~B}$ ). Simultaneous administration of the $\mathrm{CCl}_{4}$ and L-NAME had no effect on the degree of lipid peroxidation compared with $\mathrm{CCl}_{4}$ treatment alone.

NOS expression during $\mathrm{CCl}_{4}$ hepatotoxicity. eNOS and iNOS mRNA was determined by real time PCR and expressed as a ratio to GAPDH. Administration of $\mathrm{CCl}_{4}$ with or without L-NAME had no effect on eNOS expression (Fig. 4A). iNOS mRNA was not detected in any animal. eNOS and iNOS

Table 1. Real time PCR primer sequences, product size, and positive controls

\begin{tabular}{clcc}
\hline Target gene & \multicolumn{1}{c}{ Primer sequences $\left(5^{\prime}-3^{\prime}\right)$} & Size (bp) & Positive control \\
\hline eNOS & GCTTGGGATCCCTGGTATTT (F) & 85 & Brain (LPS treated) \\
GNOS & GCTTGACCCAATAGCTGCTC (R) & 95 & Brain (LPS treated) \\
GGGAGCCAGAGCAGTACAAG (F) & \multirow{2}{*}{ Liver } \\
\hline
\end{tabular}



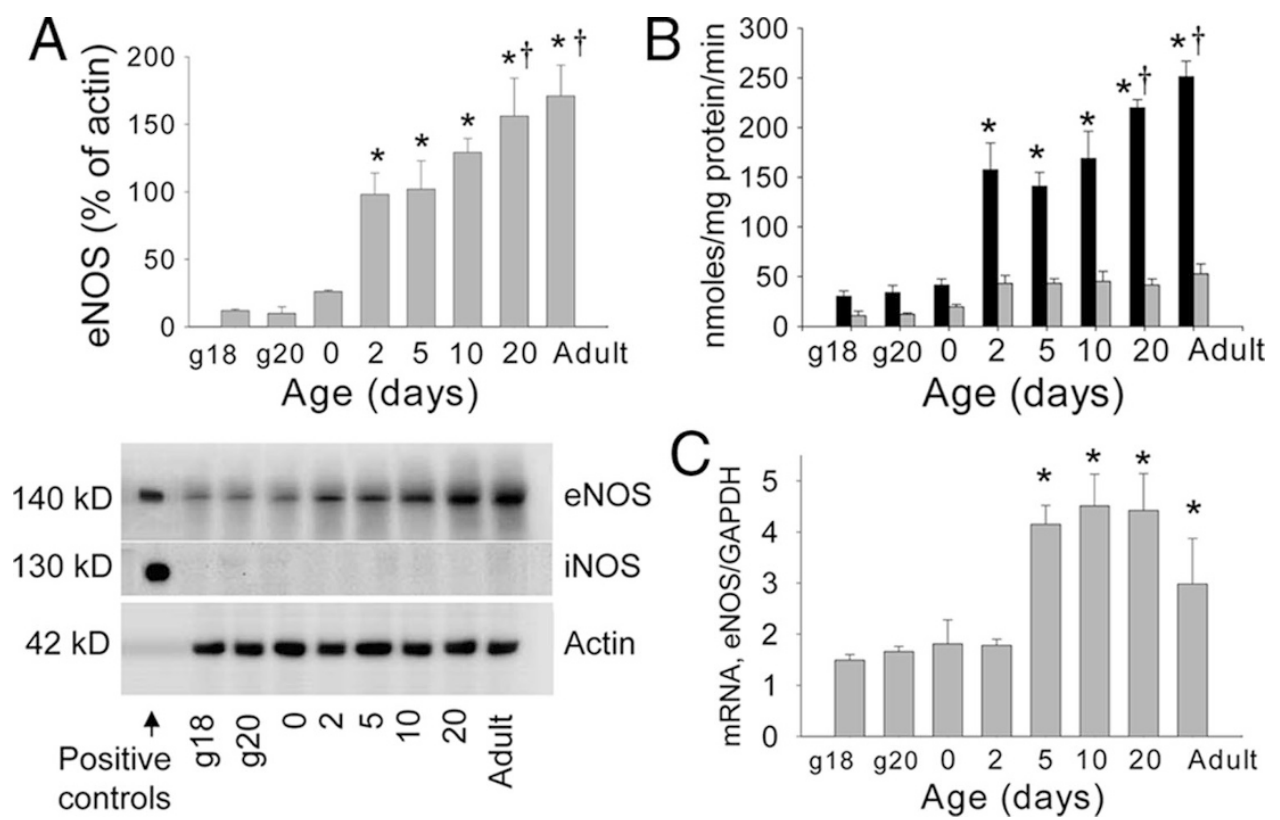

Figure 1. Developmental expression of eNOS and iNOS. (A) Semiquantitative NOS protein assay was performed by western blot. Densitometric analysis was based on three blots and standardized to actin. Values are depicted as means \pm SE expressed as a percentage of actin; $n=3$ for each time point; $* p<0.05$ compared with eNOS at $18 \mathrm{~d}$ gestation (g-18); $\dagger p<0.05$ compared with DOL-5. iNOS protein was not detectable as shown in the representative blot. (B) eNOS activity was measured with (gray) or without specific eNOS inhibitor (black) in the reaction mixture. Inhibitor was used to confirm that OD change was specific for eNOS activity. Values are means \pm SE; $n=6-8$ for each time point; $* p<0.05$ compared with $g-18 ; \dagger p<0.05$ compared with DOL-5. iNOS activity was not detectable at any age. $(C)$ Expression of eNOS mRNA as analyzed by real time PCR and standardized to GAPDH. Values are means \pm SE expressed as ratio of eNOS to GAPDH; $n=3$ (measured in triplicate) for each time point; $* p<0.05$ compared with eNOS at 18 d. iNOS mRNA was not detectable by real time PCR at any age.

proteins were determined by Western blot analysis using actin as the constitutively expressed protein. Similar to mRNA, administration of $\mathrm{CCl}_{4}$ with or without L-NAME had no effect on eNOS protein expression (Fig. $4 B$ ). iNOS protein was not detected in any animal (Fig. $4 D$ ). $\mathrm{CCl}_{4}$ administration had no effect on eNOS activity on DOL-0; however, eNOS activity increased significantly at all ages beginning on DOL-2. Coadministration of $\mathrm{CCl}_{4}$ and L-NAME had no effect on eNOS activity on DOL-0, but decreased activity below control levels in all ages from DOL-2 (Fig. 4C). iNOS activity was not detected under any conditions.

Posttranslational regulation of eNOS. There is a disconnect between eNOS expression and activity in $\mathrm{CCl}_{4}$-induced hepatotoxicity. Evidence indicates that eNOS activity can be activated by posttranslational modification mediated by phosphorylation of eNOS through the PI3k/Akt signaling pathway $(17,18)$. We examined posttranslational regulation of eNOS in our experimental model. $\mathrm{CCl}_{4}$ treatment resulted in increased phosphorylation of both Akt and eNOS (the active forms of Akt and eNOS, respectively) as determined by western blot analysis (Fig. 5A and $B$ ). Increased phosphorylation of eNOS was associated with increased eNOS activity (Fig. 6A). Treatment with wortmannin (inhibitor of PI3 K/Akt signaling) reduced eNOS activity below control values. Similarly, increased eNOS activity seen with $\mathrm{CCl}_{4}$ administration was negated by coadministration of wortmannin and $\mathrm{CCl}_{4}$ (Fig. 6A). Western blot analysis showed that wortmannin attenuated phosphorylation of Akt and eNOS both in control and $\mathrm{CCl}_{4}$-treated rats (Fig. $5 A$ and $B$ ). Co-administration of $\mathrm{CCl}_{4}$ and wortmannin potenti- ated the degree of hepatic injury compared with $\mathrm{CCl}_{4}$ alone; wortmannin alone did not cause hepatic injury (Fig. 6B).

\section{DISCUSSION}

The role of NOS in hepatic disease is complicated. The effect of NOS, mediated through production of NO, has variable, and sometimes, contradictory effects. NO can protect the liver by scavenging lipid radicals and inhibiting the lipid peroxidation chain reaction (13). Alternatively, hydroxyl radicals formed by the reaction of $\mathrm{NO}$ and superoxide anion via peroxynitrate may result in hepatotoxicity (10). NO is also important in regulating hepatic blood flow, with an indirect effect on hepatocellular injury (6). In this study, we sought to characterize the role of $\mathrm{NOS}$ in $\mathrm{CCl}_{4}$-induced hepatotoxicity in the developing liver.

Our data shows that administration of $\mathrm{CCl}_{4}$ leads to mild increase in serum ALT in the newborn rat consistent with previous studies (1-3). Administration of $\mathrm{CCl}_{4}$ led to an increase in hepatic eNOS activity with no detectable iNOS activity. These findings are in contrast to some studies showing induction of iNOS in adult rats following exposure to $\mathrm{CCl}_{4}$ $(6,13,19)$, but in agreement with other studies showing exposure to $\mathrm{CCl}_{4}$ results in eNOS activation (20) without upregulation of iNOS $(21,22)$. In this study, co-administration of $\mathrm{CCl}_{4}$ and L-NAME led to a worsening of hepatic injury, suggesting a protective role for eNOS in $\mathrm{CCl}_{4}$-induced toxicity.

Studies have shown that newborn rat is "resilient" to $\mathrm{CCl}_{4}$ toxicity $(1,3,4)$ based on serum ALT levels and lethality 


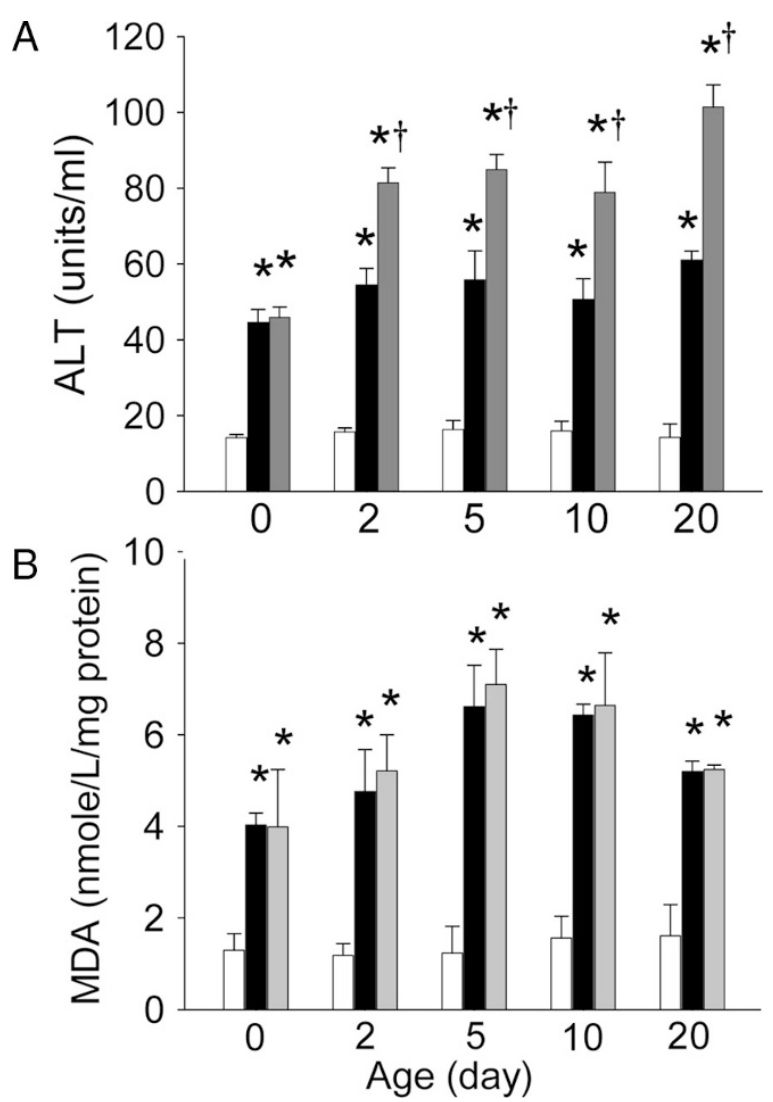

Figure 2. Liver injury and lipid peroxidation induced by $\mathrm{CCl}_{4}$ with or without L-NAME. Columns represent control (white), $\mathrm{CCl}_{4}$ (black), and $\mathrm{CCl}_{4} / \mathrm{L}-\mathrm{NAME}$ (gray) groups. (A) Liver injury was measured by ALT. Values are means $\pm \mathrm{SE} ; n=3$ each group at each time point; $* p<0.05$ compared with respective controls in each age group; $\dagger p<0.05$ compared with $\mathrm{CCl}_{4}$ treatment alone in the same age group. $(B)$ Lipid peroxidation was measured by MDA. $\mathrm{CCl}_{4}$ treatment increased MDA generation. Co-administration of $\mathrm{CCl}_{4}$ and L-NAME had no effect on the degree of lipid peroxidation. Values are means $\pm \mathrm{SE} ; n=3$ for each time point; $* p<0.05$ compared with respective controls.

compared with adult rats. Resiliency has been explained by undeveloped cytochrome P450, increased hepatocellular growth, and tissue repair mechanisms in young rats $(1,4)$. Yet, in our study, histopathological changes were present in all animals. These data are consistent with a previous study, where livers from DOL-1 rats were minimally damaged by $\mathrm{CCl}_{4}$ compared with rats at DOL-4, 10, 14, 21, and adults (23). $\mathrm{CCl}_{4}$ is bioactivated by cytochrome $\mathrm{P} 450$ to free radicals. The major isozyme for the process is cytochrome $\mathrm{P} 450$ 2E1 (24). Ontogeny studies have demonstrated that cytochrome P450 2E1 protein is not detectable in the liver of DOL-1 rat; starts to increase rapidly on DOL-3; and reaches approximately $50 \%$ of adult levels on DOL-28 (25). The ontogeny of cytochrome P450 2E1 is mirrored by hepatic damage in our and previous studies (23), where no significant histologic alterations are seen at DOL-0 and DOL- 1 but similar histologic damage is seen regardless of age after DOL-2. Lower serum ALT in $\mathrm{CCl}_{4}$-treated newborn rats does not indicate less hepatic injury compared with adult rats. Rather, the lower serum ALT in newborns could be simply because of less availability of the hepatic enzyme to leak into the blood stream during $\mathrm{CCl}_{4}$ toxicity. Supporting evidence

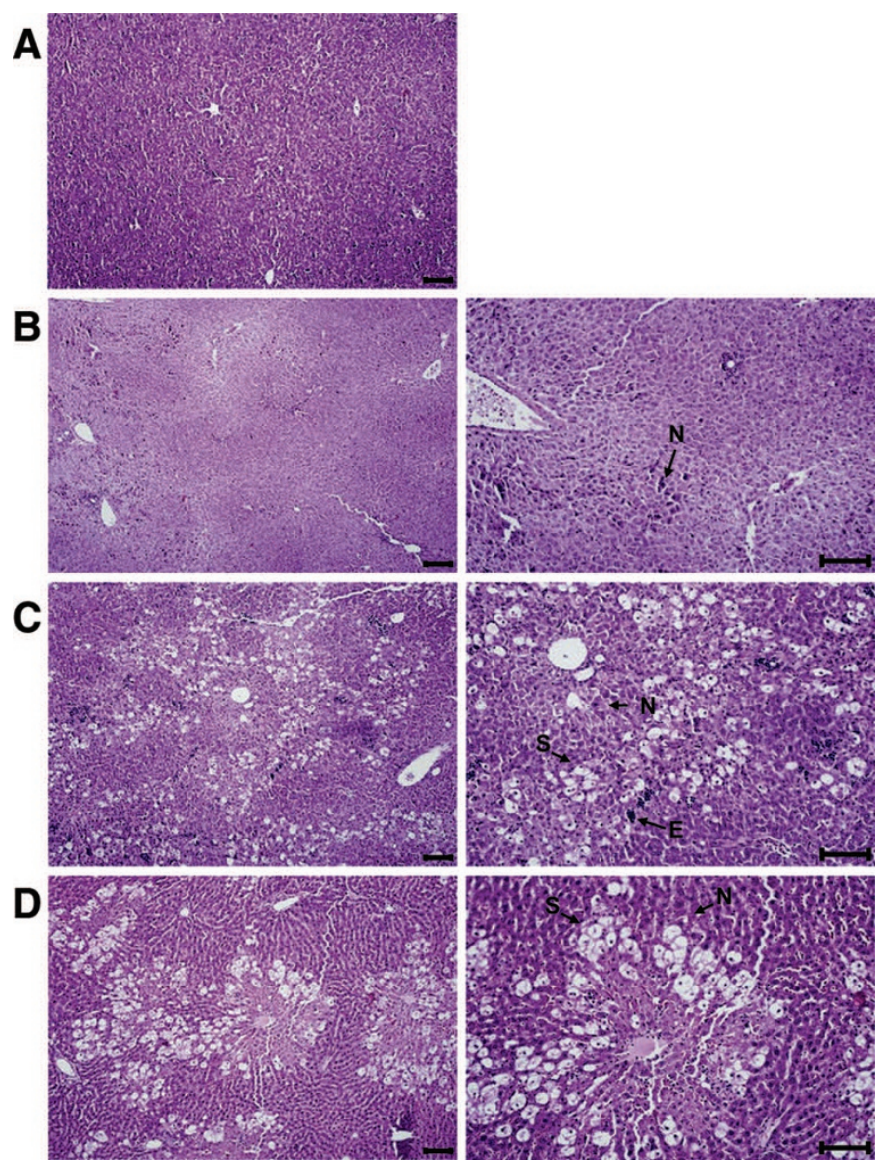

Figure 3. Histologic examination. Representative photomicrographs of liver sections from control rat $(A)$ and $\mathrm{CCl}_{4}$-treated rats: DOL-0 $(B)$, DOL-2 $(C)$, and adult $(D)$. Liver section of DOL-2 represents the histology of DOL-2, 5, 10 , and 20 since they have similar histopathological changes. $N=$ necrosis; $\mathrm{S}=$ swollen cells; $\mathrm{E}=$ extramedullary hematopoiesis. Magnifications were $100 \times$ and $200 \times$. The bar on each picture is $10 \mu \mathrm{m}$ in length.

comes from a previous study showing that hepatocellular ALT content is significantly lower in newborn than in adult rats (26).

Previous studies report mixed results when assessing inhibition of $\mathrm{NOS}$ in $\mathrm{CCl}_{4}$-induced hepatotoxicity, particularly the effect on peroxidation. Administration of a nonselective NOSinhibitor (L-NAME) increases lipid peroxidation following exposure to $\mathrm{CCl}_{4}$, suggesting $\mathrm{NO}$ plays a protective role in $\mathrm{CCl}_{4}$ intoxication through interaction with reactive oxygen species (ROS) (13). Conversely, a selective iNOS inhibitor (L-NIL) inhibited peroxidation following $\mathrm{CCl}_{4}$ administration suggesting that $\mathrm{NO}$ plays a deleterious role in $\mathrm{CCl}_{4}$ intoxication in adult rats (19). We measured hepatic tissue MDA following $\mathrm{CCl}_{4}$ exposure showing that, while lipid peroxidation occurs, it is not altered by co-administration of $\mathrm{CCl}_{4}$ and L-NAME despite increased hepatic injury. This data would suggest that in this model NO does not participate in lipid peroxidation through interaction with ROS or lipid peroxyl groups.

An alternative explanation is that eNOS offers protection from acute $\mathrm{CCl}_{4}$-induced toxicity by a mechanism unrelated to ROS production, such as changes in hepatic blood flow. Previous data have shown that acute $\mathrm{CCl}_{4}$ toxicity results in increased hepatic blood flow, both in the portal vein and 

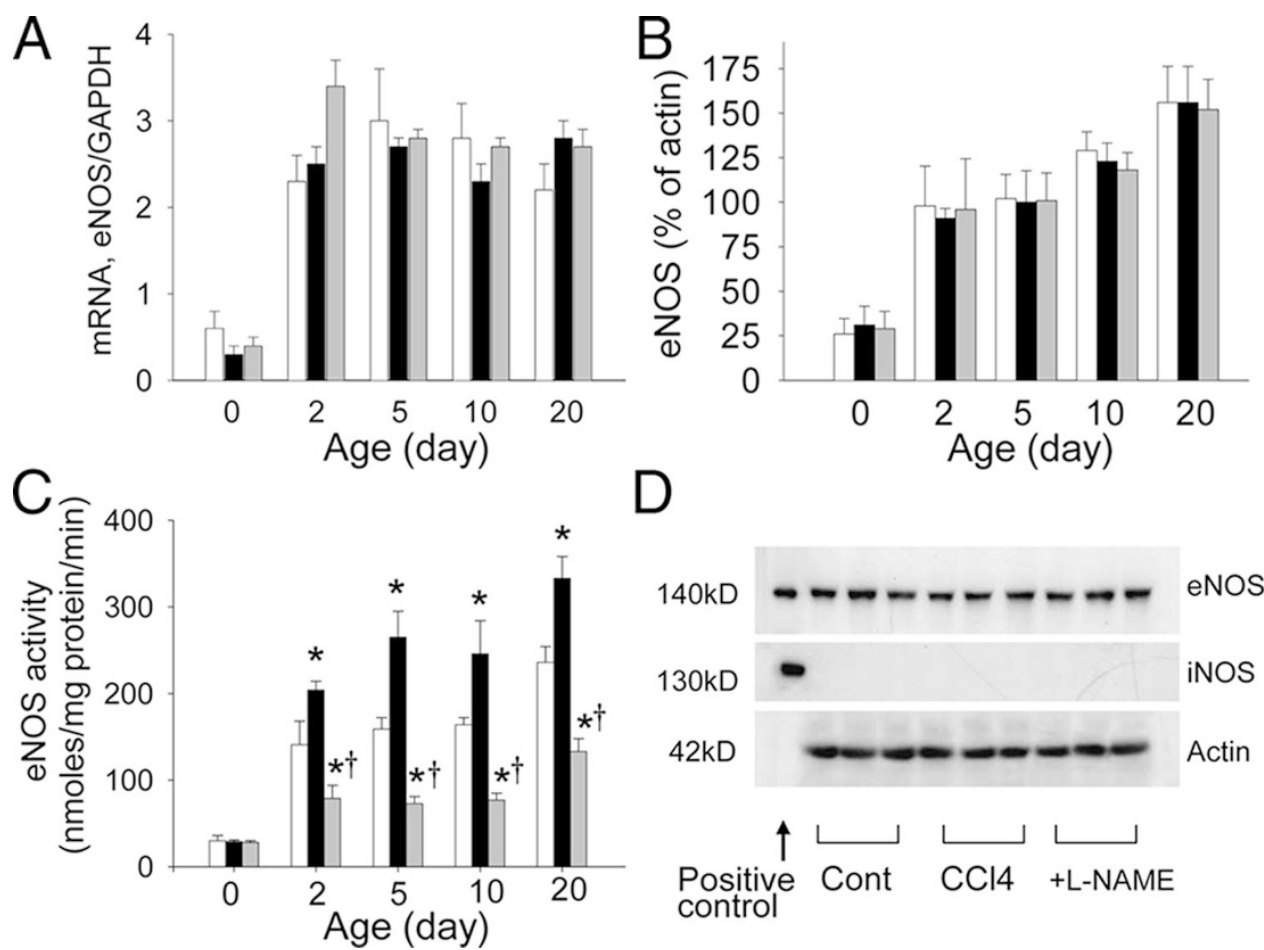

Figure 4. Expression of eNOS in the liver of newborn rats treated with $\mathrm{CCl}_{4}$ with or without L-NAME. Columns represent control (white), $\mathrm{CCl}_{4}$ (black), and

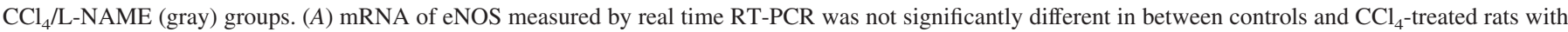
or without L-NAME in the same age groups. eNOS mRNA was standardized to GAPDH. Values are means \pm SE expressed as ratio of eNOS to GAPDH; $n=3$ (measured in triplicate). (B) Western blot showed no significant difference in eNOS protein expression between controls and $\mathrm{CCl}_{4}$-treated rats with or without L-NAME in the same age groups. Values are depicted as means \pm SE expressed as a percentage of actin; $n=3$ for each time point. $(C)$ eNOS activity was significantly increased in $\mathrm{CCl}_{4}$-treated rats as compared with respective control. Co-administration of L-NAME with $\mathrm{CCl}_{4}$ decreased eNOS activity below control levels. Values are means $\pm \mathrm{SE} ; n=3$ for each time point (measured in triplicate). (D) iNOS was not induced in $\mathrm{CCl}_{4}$-treated rats with or without L-NAME as shown in the representative blot of DOL-20 rats. ${ }^{*} p<0.05$ compared with respective control in each age group; $\dagger p<0.05$ compared with $\mathrm{CCl}_{4}$ treatment alone.
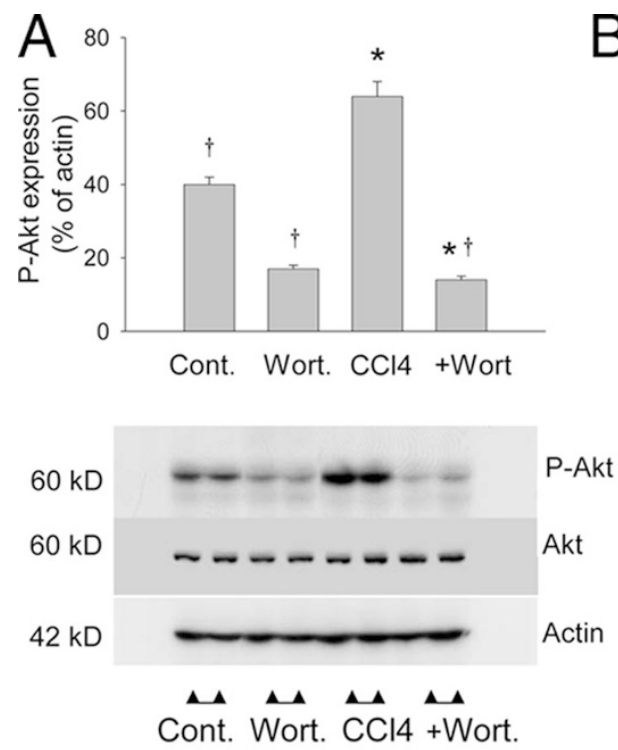
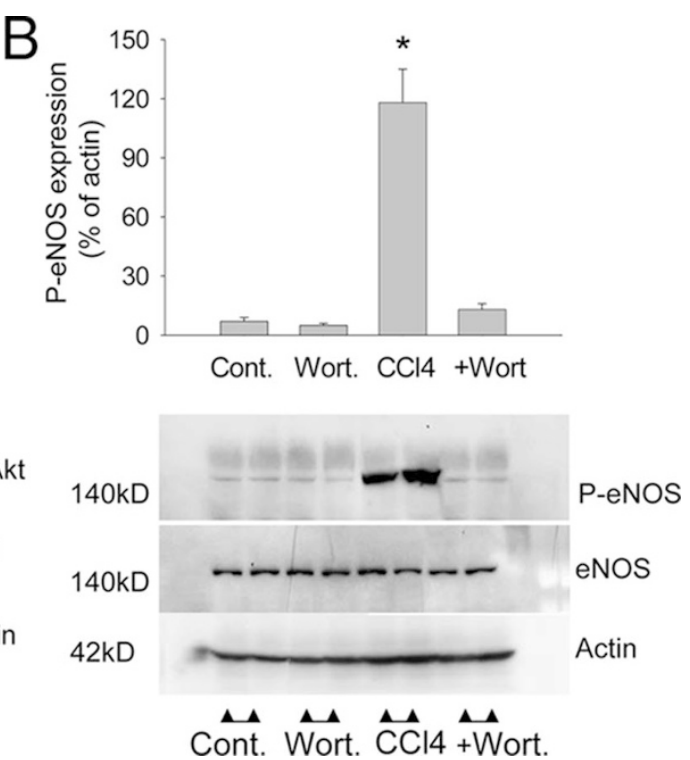

Figure 5. Determination of phosphorylation of Akt and eNOS by western blot. There was an increase in phosphorylation of both Akt $(A)$ and eNOS $(B)$ in the liver of $\mathrm{CCl}_{4}$-treated DOL-20 rats. Administration of wortmannin (Wort) significantly reduced the phosphorylation both in control (Cont) and $\mathrm{CCl}_{4}$-treated animals. Values are means $\pm \mathrm{SE}$ expressed as a percentage of actin; $n=3 ; * p<0.05$ compared with respective control; $\dagger p<0.05$ compared with $\mathrm{CCl}_{4}$ treatment alone. A representative blot is shown. hepatic artery $(5,6)$. Increase in hepatic artery blood flow is mediated by increased NO production via up-regulation of NOS, resulting in reduced hepatic damage. Inhibition of NOS activity dampens hepatic artery flow exacerbating hepatic damage (6). NO has also been shown to improve hepatic microvascular perfusion through reducing neutrophil accumulation and platelet aggregation (27). Beneficial effects of in- creased hepatic blood flow in $\mathrm{CCl}_{4}$-induced injury may be related to improved oxygen delivery to the damaged liver (6). Inflammation results in increased arterial blood flow due to increased oxygen demand for synthesis of DNA and proteins needed for tissue repair in hepatic regeneration (28). In $\mathrm{CCl}_{4}$ toxicity, increased hepatic arterial blood flow provides greater oxygen supply to promote liver regeneration following acute 

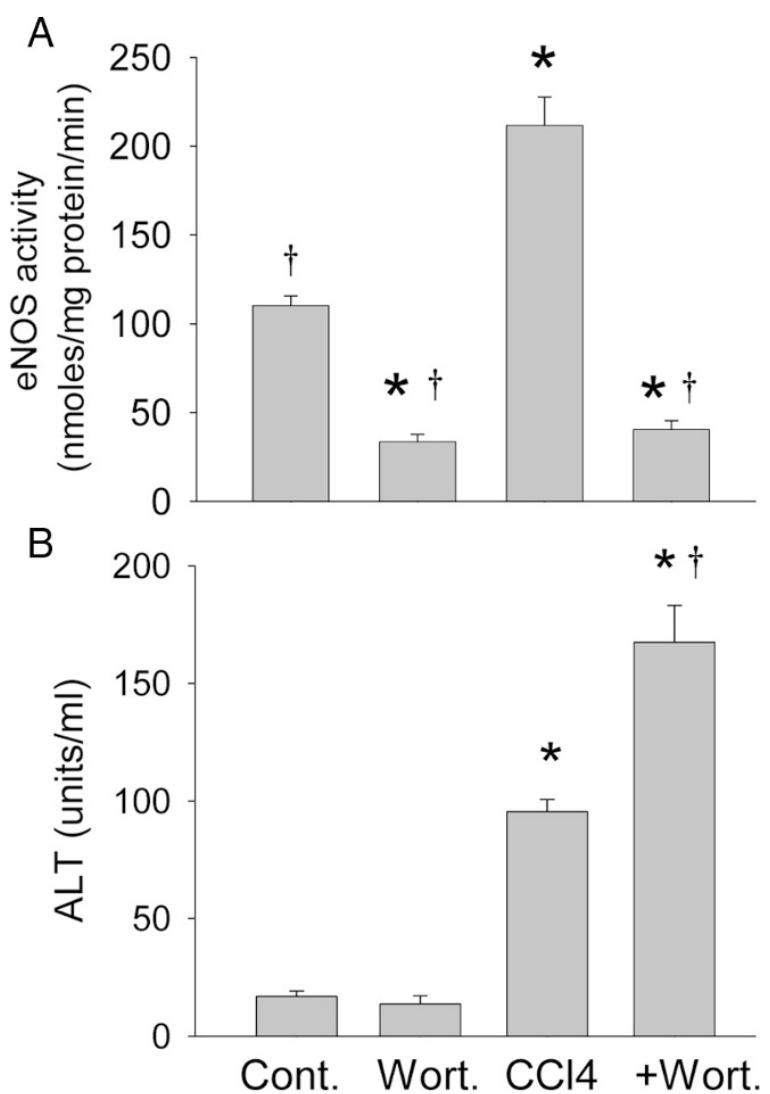

Figure 6. eNOS activity and liver function of newborn rats treated with $\mathrm{CCl}_{4}$ with or without wortmannin. (A) eNOS activity was significantly increased in $\mathrm{CCl}_{4}$-treated rats compared with controls (Cont). Administration of wortmannin (Wort) reduced eNOS activity both in control and $\mathrm{CCl}_{4}$-treated animals. Values are means $\pm \mathrm{SE} ; n=3$ for each time point (measured in triplicate). (B) Liver function as measured by ALT showed that wortmannin (Wort) potentiated the degree of hepatic injury compared with $\mathrm{CCl}_{4}$ alone. Administration wortmannin alone did not cause hepatic injury. Values are means \pm $\mathrm{SE} ; n=3$ (measured in triplicate). ${ }^{*} p<0.05$ compared with control (Cont); $\dagger p<0.05$ compared with $\mathrm{CCl}_{4}$ treatment alone.

hepatic injury (6). Although the exact protective mechanism afforded against $\mathrm{CCl}_{4}$ toxicity by increased hepatic artery blood flow remains to be determined, further study on hepatic hemodynamic changes in $\mathrm{CCl}_{4}$ toxicity will strengthen the current findings.

The principle control of hepatic blood flow under normal conditions is at the level of the sinusoid and is mediated by eNOS (8). In newborn rats, eNOS mRNA, protein, and activity increase in parallel reaching adult levels by $20 \mathrm{~d}$ of life (9). Immunohistochemical staining reveals that eNOS is present in the sinusoids at birth, albeit in low amounts and scattered across the lobule. Sinusoidal eNOS increases in intensity and distribution with age, reaching an adult pattern by $20 \mathrm{~d}$ of life (9). There is also data showing that the hepatic artery contributes more to sinusoidal blood flow maintenance in the immature liver than in the adult liver (7). At baseline, the hepatic artery is under the control of NOS. Supportive evidence includes vasoconstriction, and resulting increased hepatic artery resistance, following inhibition of NOS by L-NAME and partial reversal of vasoconstriction following administration of L-arginine (29). These findings may help explain why, despite paucity of eNOS in the sinusoids of the newborn rat,
eNOS offers protection in $\mathrm{CCl}_{4}$ toxicity. Additionally, the protective effect of $\mathrm{NO}$ may be related to its anti-apoptosis activity and protection against cytokine mediated cell death (30).

In this model of hepatotoxicity, eNOS activity was increased after exposure to $\mathrm{CCl}_{4}$, without changes in mRNA and protein levels. This increase in eNOS activity might be associated with the posttranslational modification of eNOS due to phosphorylation mediated by Akt, a down stream effector in the PI3 K/Akt signaling pathway. Previous studies have shown that Akt directly phosphorylates eNOS, activating it to produce NO both in transfected COS-7 cells and cultured endothelial cells (17). Akt has no effect on iNOS or nNOS (17), making any contribution of these forms of NOS unlikely in our model. Activation of Akt is dependent on the degree of shear force stress, with direct correlation between increased stress and increased Akt phosphorylation (18). In the current study, treatment of newborn rats with $\mathrm{CCl}_{4}$ resulted in increased phosphorylation of Akt and eNOS associated with increased eNOS activity. Administration of the PI3 K inhibitor wortmannin resulted in reduction of Akt and eNOS phosphorylation both in control and $\mathrm{CCl}_{4}$-treated animals. Coadministration of $\mathrm{CCl}_{4}$ and wortmannin, led to a significant decrease in eNOS activity and increase in hepatic damage. Thus, posttranslational eNOS activation is responsible for increased eNOS activity following $\mathrm{CCl}_{4}$ exposure.

In conclusion, we have confirmed that newborn animals exposed to $\mathrm{CCl}_{4}$ have hepatic injury, which is associated with increased levels of eNOS activity. Co-administration of $\mathrm{CCl}_{4}$ and L-NAME results in worsening of hepatic damage, suggesting a protective role for eNOS in this model. We propose that the protective effect of eNOS is accomplished by its activation through the posttranslational modification via PI3 K/Akt signaling pathway.

\section{REFERENCES}

1. Cai Z, Mehendale HM 1993 Resiliency to amplification of carbon tetrachloride hepatotoxicity by chlordecone during postnatal development in rats. Pediatr Res 33:225-232

2. Sipes IG, Krishna G, Gillette JR 1977 Bioactivation of carbon tetrachloride, chloroform and bromotrichloromethane: role of cytochrome P-450. Life Sci 20:15411548

3. Dawkins MJ 1963 Carbon tetrachloride poisoning in the liver of the newborn rats. J Pathol Bacteriol 85:189-196

4. Dalu A, Cronin GM, Lyn-Cook BD, Mehendale HM 1995 Age-related differences in TGF-alpha and proto-oncogene's expression in rat liver after a low dose of carbon tetrachloride. J Biochem Toxicol 10:259-264

5. Lautt WW, Plaa GL 1974 Hemodynamic effects of CCl4 in the intact liver of the cat. Can J Physiol Pharmacol 52:727-735

6. Tanaka N, Tanaka K, Nagashima Y, Kondo M, Sekihara H 1999 Nitric oxide increases hepatic artery blood flow in rats with carbon tetrachloride-induced acute hepatic injury. Gastroenterology 117:173-180

7. Drugas GT, Chun KE, Miescher EA, Clemens MG 1993 Maturational differences in hepatic microhemodynamics in rats. J Surg Res 54:246-253

8. Shah V, Haddad FG, Garcia-Cardena G, Frangos JA, Mennone A, Groszmann RJ, Sessa WC 1997 Liver sinusoidal endothelial cells are responsible for nitric oxide modulation of resistance in the hepatic sinusoids. J Clin Invest 100:2923-2930

9. Nowicki MJ, Shi D, Cai Z, Bishop PR, May WL 2003 Developmental expression of endothelial nitric oxide synthase (eNOS) in the rat liver. Pediatr Res 54:732-738

10. Beckman JS, Koppenol WH 1996 Nitric oxide, superoxide, and peroxynitrite: the good, the bad, and ugly. Am J Physiol 271:C1424-C1437

11. Nanji AA, Greenberg SS, Tahan SR, Fogt F, Loscalzo J, Sadrzadeh SM, Xie J, Stamler JS 1995 Nitric oxide production in experimental alcoholic liver disease in the rat: role in protection from injury. Gastroenterology 109:899-907

12. Kim YM, Bergonia H, Lancaster JR 1995 Nitrogen oxide-induced autoprotection in isolated rat hepatocytes. FEBS Lett 374:228-232

13. Zhu W, Fung PC 2000 The roles played by crucial free radicals like lipid free radicals, nitric oxide, and enzymes NOS and NADPH in $\mathrm{CCl}(4)$-induced acute liver injury of mice. Free Radic Biol Med 29:870-880 
14. Wenger FA, Kilian M, Mautsch I, Jacobi CA, Steiert A, Peter FJ, Guski H, Schimke I, Muller JM 2001 Influence of octreotide on liver metastasis and hepatic lipid peroxidation in BOP-induced pancreatic cancer in Syrian hamsters. Pancreas 23:266-272

15. Rozen S, Skaletsky HJ 2000 Primer3 on the www for general users and for biologist programmers. In: Krawetz S, Misener S (eds) Bioinformatics Methods and Protocols: Methods in Molecular Biology. Humana Press, Totowa, pp 365-386

16. Kelley TJ, Drumm ML 1998 Inducible nitric oxide synthase expression is reduced in cystic fibrosis murine and human airway epithelial cells. J Clin Invest 102:12001207

17. Fulton D, Gratton JP, McCabe TJ, Fontana J, Fujio Y, Walsh K, Franke TF, Papapetropoulos A, Sessa WC 1999 Regulation of endothelium-derived nitric oxide production by the protein kinase Akt. Nature 399:597-601

18. Dimmeler S, Fleming I, Fisslthaler B, Hermann C, Busse R, Zeiher AM 1999 Activation of nitric oxide synthase in endothelial cells by Akt-dependent phosphorylation. Nature 399:601-605

19. Tipoe GL, Leung TM, Liong E, So H, Leung KM, Lau TY, Tom WM, Fung ML, Fan ST, Nanji AA 2006 Inhibitors of inducible nitric oxide (NO) synthase are more effective than an NO donor in reducing carbon-tetrachloride induced acute liver injury. Histol Histopathol 21:1157-1165

20. Stumm MM, D’Orazio D, Sumanovski LT, Martin PY, Reichen J, Sieber CC 2002 Endothelial, but not the inducible, nitric oxide synthase is detectable in normal and portal hypertensive rats. Liver 22:441-450
21. Moreno MG, Muriel P 2006 Inducible nitric oxide synthase is not essential for the development of fibrosis and liver damage induced by $\mathrm{CCl} 4$ in mice. J Appl Toxicol 26:326-332

22. Martin PY, Xu DL, Niederberger M, Weigert A, Tsai P, St John J, Gines P, Schrier RW 1996 Upregulation of endothelial constitutive NOS: a major role in the increased NO production in cirrhotic rats. Am J Physiol 270:F494-F499

23. Cagen SZ, Klaasen CD 1980 Carbon tetrachloride-induced hepatotoxicity: studies in developing rats and protection by zinc. Fed Proc 39:3124-3128

24. Weber LW, Boll M, Stampfl A 2003 Hepatotoxicity and mechanism of action of haloalkanes: carbon tetrachloride as a toxicological model. Crit Rev Toxicol 33:105136

25. Elbarbry FA, McNamara PJ, Alcorn J 2007 Ontogeny of hepatic CYP1A2 and CYP2E1 expression in rat. J Biochem Mol Toxicol 21:41-50

26. Dalu A, Mehendale HM 1996 Efficient tissue repair underlies the resiliency of postnatally developing rats to chlordecone $+\mathrm{CCl} 4$ hepatotoxicity. Toxicology 111:29-42

27. Clemens MG 1999 Nitric oxide in liver injury. Hepatology 30:1-5

28. Ozawa K 1990 Hepatic function and liver resection. J Gastroenterol Hepatol 5:296-309

29. Ayuse T, Brienza N, Revelly JP, Boitnott JK, Robotham JL 1995 Role of nitric oxide in porcine liver circulation under normal and endotoxemic conditions. J Appl Physiol 78:1319-1329

30. Hon WM, Lee KH, Khoo HE 2002 Nitric oxide in liver diseases: friend, foe, or just passerby? Ann N Y Acad Sci 962:275-295 